\title{
National Trends in the Use of Targeted Therapy and Immunotherapy in the Up Front Management of Glioblastoma
}

\author{
Richard White', Stephen Abel ${ }^{2}$, Shaakir Hasan², Vivek Verma ${ }^{2}$, Tulika Ranjan ${ }^{3}$, Stephen M. Karlovits², Rodney E \\ Wegner ${ }^{2 *}$
}

'Allegheny Health Network, Department of Internal Medicine, Pittsburgh, PA, USA

${ }^{2}$ Allegheny Health Network Cancer Institute, Division of Radiation Oncology, Pittsburgh, PA, USA

${ }^{3}$ Allegheny Health Network Cancer Institute, Division of Neuro Oncology, Pittsburgh, PA, USA

Article Info

\section{Article Notes}

Received: April 10, 2019

Accepted: May 7, 2019

\section{${ }^{*}$ Correspondence:}

Dr. Rodney E. Wegner, Allegheny Health Network Cancer Institute, Division of Radiation Oncology, Allegheny Genera Hospital, Level 02, 320 E. North Avenue, Pittsburgh, PA 15212, USA; Phone No: 412-359-3400; Fax No: 412-3593171; Email: Rodney.Wegner@ahn.org.

C 2019 Wegner RE. This article is distributed under the terms of the Creative Commons Attribution 4.0 International License.

\section{ABSTRACT}

Glioblastoma (GBM) carries an abysmal prognosis. Current standard of care involves an aggressive multimodality approach including surgical resection followed by adjuvant chemoradiation. Despite this approach, overall survival remains poor and treatment approaches continue to evolve. Given the successes of immunotherapy in other disease sites, implementation in GBM management may improve outcomes. We conducted this retrospective National Cancer Database (NCDB) study to analyze treatment trends and outcomes from 2004-2015 regarding immunotherapy for GBM and queried for patients diagnosed between 2004-2015 with GBM and excluded patients treated without surgery, extracranial radiation, or chemotherapy as well as those lost to follow up.

Of the 39,317 eligible patients in this study, 511 were treated with immunotherapy and 38,806 lack thereof. Median overall survival for all patients was 15 months with a 2 and 5 year survival rate of $29 \%$ and $8 \%$, respectively. Factors positively influencing delivery of immunotherapy included younger age, higher income, facility location in a metropolitan location, greater distance to the treatment facility, treatment at an academic facility, treatment outside of the years 2007 to 2009, and Caucasian race. On propensity matched analysis, survival was 18 months and 17 months with and without immunotherapy, respectively $(p=0.15)$. Higher comorbidity, lower income, and male gender predicted for worse survival.

The results of the NCDB analysis showed an initial decrease and then increase in the use of immunotherapy in the management of GBM. Propensitymatched analyses did not show an overall survival benefit.

\section{Background}

Glioblastoma (GBM) is the most common malignant central nervous system (CNS) tumor in adults, accounting for nearly half of all annual primary malignant CNS diagnoses. Despite advances in diagnostic technologies and therapeutic techniques, relative 2 and 5-year survival rates are approximately 15\% and $5 \%$, respectively ${ }^{1}$. Since the landmark 2005 study by Stupp et al, the current standard of care remains maximal safe surgical resection followed by radiotherapy with concurrent and adjuvant temozolomide ${ }^{2}$. Nevertheless, outcomes remain poor despite the modest improvement in survival attributed to the addition of temozolomide. As such, more efficacious alternatives are areas of ongoing investigation. Trials have been conducted showing relative risk reduction with local chemotherapies ${ }^{3}$ and CT-guided interstitial high-dose-radiation brachytherapy ${ }^{4}$ along with ongoing others that 
show promise. One such recent advancement, and now considered to be standard of care, tumor-treating fields, known to most as the Optune device, a treatment modality which applies low-intensity alternating electric fields to interfere with GBM mitotic cell division has been shown to significantly improve overall survival ${ }^{5}$.

Over the last decade, use of targeted and immunotherapy (IMT) compounds has resulted in improved oncologic outcomes in various advanced malignancies, thus revolutionizing management. Interestingly, a mounting body of evidence in the metastatic setting suggests that many immunotherapeutic agents have CNS activity. Promise has been shown in the ability of immunemodulating antibodies to cross the blood brain barrier, particularly with ipilimumab therapy for brain metastasis from malignant melanoma and pembrolizumab for nonsmall cell lung cancer with brain metastasis ${ }^{6}$. Conversely, the role of IMT in primary CNS malignancies, specifically GBM, is not well established. Unfortunately, results from CheckMate-143, a randomized phase 3 trial comparing the efficacy and safety between nivolumab and bevacizumab therapy for GBM first recurrence, failed to show improved overall survival with the use of nivolumab over bevacizumab. Additionally, a small retrospective series examining the use of pembrolizumab in recurrent primary intracranial malignancies reported no clinical benefit ${ }^{7}$, however, data regarding the upfront use of IMT in GBM is nonexistent. As a result, we attempted to address this knowledge gap through analysis of a large, contemporary national database comparing practice patterns and outcomes in patients with GBM treated with and without upfront IMT.

\section{Methods}

The methods for performing an analysis of the National Cancer Database (NCDB) have been described previously ${ }^{8-13}$. We conducted a retrospective review using de-identified data from the NCDB; therefore the study was exempt from Institutional Review Board oversight. Jointly maintained by the American Cancer Society and the American College of Surgeons, the NCDB encompasses approximately 70\% of newly diagnosed malignancies each year across the United States. We queried the NCDB for patients diagnosed between 2004-2015 with GBM, excluding patients treated without surgery, extracranial radiation, or chemotherapy. Patients with less than 2 month follow up were excluded to account for immortal time bias. Figure 1 outlines the patient selection process. These patients (age range: 18-90) were particularly chosen for as they are those that received the currently recommended and maximum therapy allowing them the highest potential benefit from treatment. The resultant patient group was then split into those with IMT incorporated into their treatment regimen and those not. of note, targeted therapies, for instance becavizumab, as

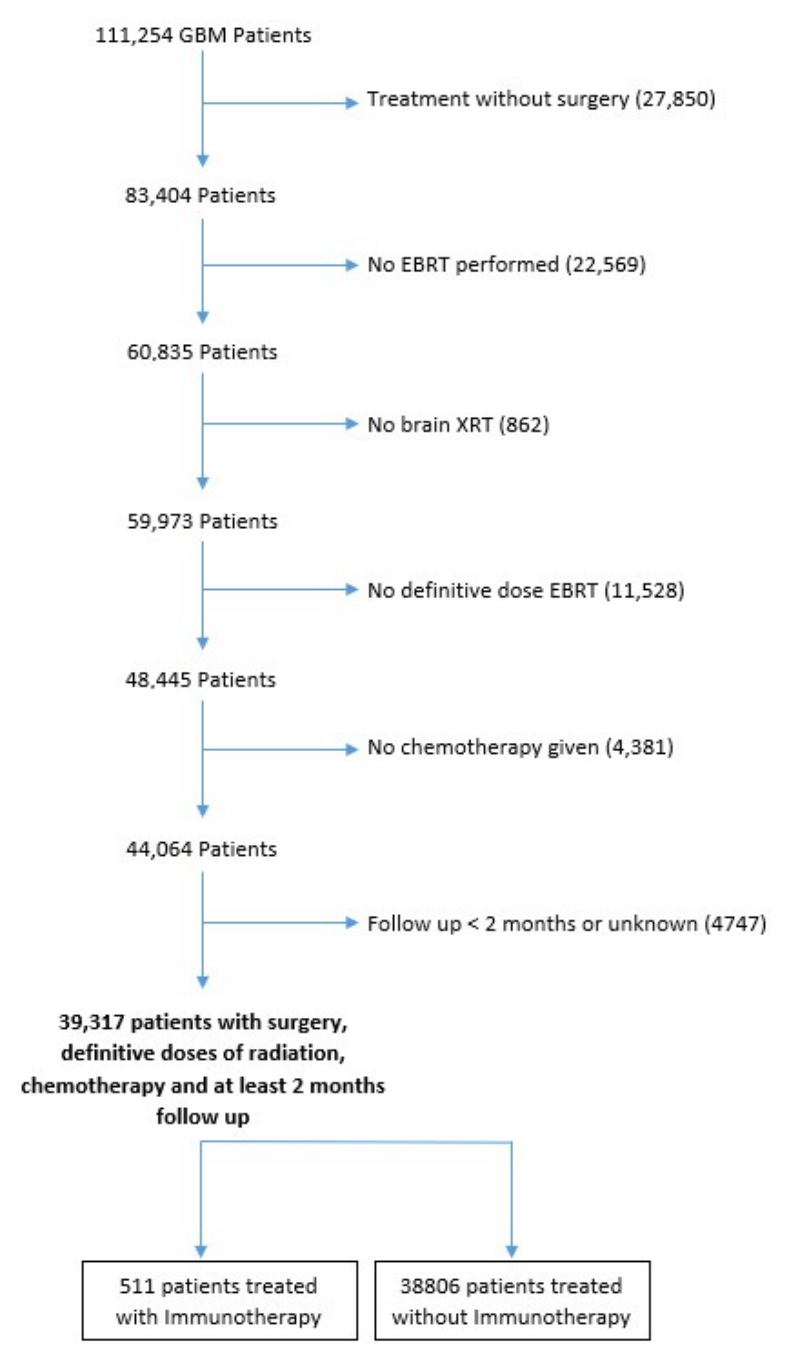

Figure 1: Selection criteria for study eligibility.

well as tumor vaccines, monoclonal antibodies and other such compounds are listed as IMT in the NCDB.

Race was divided into three broad categories including Caucasian, African American, or other. Comorbidity was quantified using the Charlson/Deyo comorbidity index ${ }^{14}$. Socioeconomic data in the patients' residence census tract were provided as quartiles of the percentage of persons with less than a high school education and median household income. The facility type was assigned according to the Commission on Cancer accreditation category. Locations were assigned based on data provided by the US Department of Agriculture Economic Research Service. Insurance status is documented in the NCDB as it appears on the admission page. The American College of Surgeons and the Commission on Cancer have not verified and are not responsible for the analytic or statistical methodology employed, or the conclusions drawn from these data by the investigator.

Data were analyzed using Medcalc Version 18 (Ostend, Belgium). Summary statistics are presented for 
discrete variables. Chi-squared testing compared patient, treatment, and disease-related characteristics between the two treatment groups. Overall survival was calculated in months from time of diagnosis to date of last contact or death. Kaplan-Meier curves were used to calculate cumulative probability of survival ${ }^{15}$. Log-rank statistics were used to test for significant differences in the cumulative proportions across groups. A Cox proportional hazards model was used for multivariable survival analysis ${ }^{16}$. Due to the large nature of the dataset, factors significant on univariable Cox regression were entered using a stepwise backward elimination process. Adjusted hazard ratios and 95\% confidence intervals are reported, using an alpha level of 0.05 to indicate statistical significance.

Propensity score-adjusted survival analysis was used to account for indication bias due to lack of randomization between patients receiving IMT and those not ${ }^{17}$. Multivariable logistic regression was used to calculate a propensity score indicative of the conditional probability regarding receipt of IMT. The propensity model included observable variables associated with treatment selection on multivariable logistic regression. A Cox proportional hazards model was then constructed incorporating the propensity score, but also excluding factors included in the propensity score calculation to avoid overcorrection. The assumption of balance was further validated by stratifying the data into propensity score-based quintiles, and confirming that the difference in propensity score mean per quintile was less than 0.10 .

\section{Results}

We identified 39,317 eligible patients, of which, 511 patients received IMT as part of their initial treatment after surgical resection in conjunction with chemoradiation. Table 1 displays patient characteristics associated with the entire cohort. In 2007 to 2009, upfront IMT use showed a substantial drop off and then rebound in 2010 (Figure 2). Predictors of IMT use included: younger age, higher income, metropolitan location, greater distance to treatment facility, treatment at an academic facility, treatment outside 2007 to 2009, and Caucasian race (Table 2). The median follow up for the entire group was 15 months (range: 2-155 months). Median follow up in the IMT cohort was 16.8 months (range: 2-146), while median follow up for patients who did not receive IMT was 15 (range: 2-155). Median overall survival was 15 months for all patients, with a 2 year overall survival of $29 \%$ and 5 year survival of $8 \%$. In all patients, median overall survival was 18 months with IMT, compared to 15 months without $(\mathrm{p}<0.0001)$ (Figure 3$)$. On multivariable analysis, increased age, male sex, private insurance, higher comorbidity score, Caucasian race, treatment before 2006, lower income, receipt of treatment at a community cancer program and less education predicted for poorer overall
Table 1. Baseline Patient Characteristics.

\begin{tabular}{|c|c|}
\hline \multicolumn{2}{|c|}{ Patient and Treatment Characteristics $(\mathrm{N}=39,317)$} \\
\hline Characteristic & No. (\% or range) \\
\hline \multicolumn{2}{|l|}{ Demographics } \\
\hline \multicolumn{2}{|l|}{ Sex } \\
\hline Male & $23371(59.4)$ \\
\hline Female & $15946(40.6)$ \\
\hline \multicolumn{2}{|l|}{ Age } \\
\hline Median & $61(18-90)$ \\
\hline$<61$ & $19443(49.5)$ \\
\hline$\geq 61$ & $19874(50.5)$ \\
\hline \multicolumn{2}{|l|}{ Race } \\
\hline White & $36031(91.6)$ \\
\hline African American & $1965(5.0)$ \\
\hline Other/Unknown & $1321(3.4)$ \\
\hline \multicolumn{2}{|l|}{ Comorbidity score } \\
\hline 0 & $29793(75.8)$ \\
\hline 1 & $6094(15.5)$ \\
\hline$\geq 2$ & $3430(8.7)$ \\
\hline \multicolumn{2}{|l|}{ Insurance } \\
\hline Not insured & $1321(3.4)$ \\
\hline Government & $16198(41.2)$ \\
\hline Private payer & $21320(54.2)$ \\
\hline Unknown & $478(1.2)$ \\
\hline \multicolumn{2}{|l|}{ Treatment facility type } \\
\hline Community cancer program & $1925(4.9)$ \\
\hline Comprehensive community cancer program & $14155(36.0)$ \\
\hline Academic/research program & $21020(53.5)$ \\
\hline Unknown & $2217(5.6)$ \\
\hline \multicolumn{2}{|l|}{ Treatment facility location } \\
\hline Metro counties & $31291(79.6)$ \\
\hline Urban counties & $5694(14.5)$ \\
\hline Rural counties & $717(1.8)$ \\
\hline Unknown & $1615(4.1)$ \\
\hline \multicolumn{2}{|l|}{ Income, US dollars } \\
\hline$<38,000$ & $5055(12.9)$ \\
\hline $\begin{array}{l}38,000-47,999 \\
48000-62,999\end{array}$ & $\begin{array}{c}8384(21.3) \\
10757(27.4)\end{array}$ \\
\hline $\begin{array}{l}>63,000 \\
\text { Unknown }\end{array}$ & $\begin{array}{c}14284(36.3) \\
837(2.1)\end{array}$ \\
\hline \multicolumn{2}{|l|}{ Distance to treatment facility, miles } \\
\hline$\leq 12$ & $18970(48.2)$ \\
\hline $\begin{array}{l}>12 \\
\text { Unknown }\end{array}$ & $\begin{array}{c}19524(49.7) \\
823(2.1)\end{array}$ \\
\hline \multicolumn{2}{|l|}{ Year of Diagnosis } \\
\hline $2004-2006$ & $7975(20.3)$ \\
\hline $2007-2009$ & $10099(25.7)$ \\
\hline $2010-2012$ & $12108(30.8)$ \\
\hline $2013-2014$ & 9135 (23.2) \\
\hline
\end{tabular}

LEGEND: No, number. CTX, chemotherapy

survival as shown in Table 3. As described in the methods, a logistic regression was used to generate a propensity score. The logistic regression model included age, facility type, education level, insurance type, location, race, and year group. Multivariable analysis with propensity score 


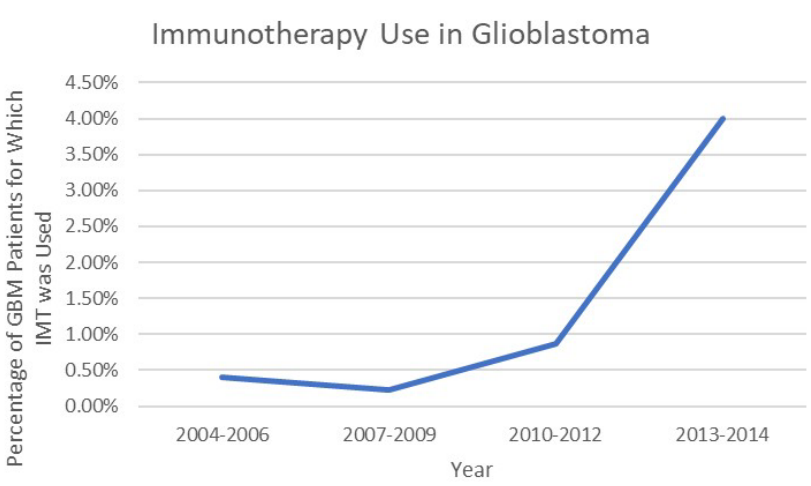

Figure 2: National trends in immunotherapy use by year.

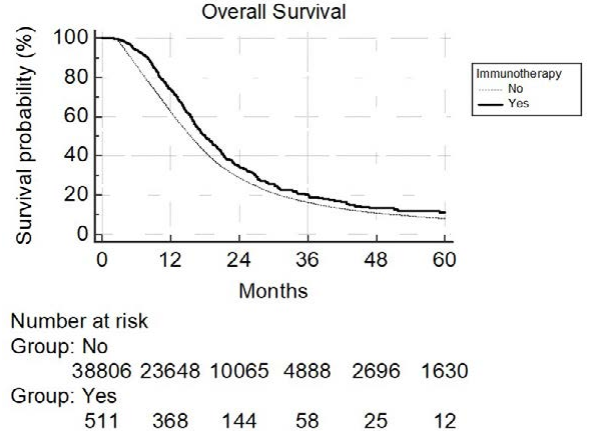

Figure 3: Overall survival with immunotherapy use on univariable analysis. Median OS: 18 vs 15 mo. ( $\mathrm{P}<0.0001) .2$ year OS: $28 \%$ vs $29 \%$. 5 year OS: $8 \%$ vs $8.1 \%$.

Table 2. Comparative Use of Immunotherapy in Management of GBM by Baseline Characteristic

\begin{tabular}{|c|c|c|c|c|c|}
\hline Characteristics & $\begin{array}{l}+\mathrm{IMT} \\
\mathrm{N}=511\end{array}$ & $\begin{array}{c}-\mathrm{IMT} \\
\mathrm{N}=38806\end{array}$ & OR & $95 \% \mathrm{Cl}$ & $P$ value \\
\hline & & & - & - & - \\
\hline \multicolumn{6}{|l|}{ Gender } \\
\hline Male & 306 (59.9) & 23065 (59.4) & 1 & Reference & \\
\hline Female & $205(40.1)$ & $15741(40.6)$ & 1.02 & $0.85-1.22$ & 0.84 \\
\hline \multicolumn{6}{|l|}{ Age } \\
\hline$<61$ & $305(49.6)$ & $19138(46.5)$ & 1 & Reference & \\
\hline$\geq 61$ & $206(50.4)$ & $19668(53.5)$ & 1.52 & $1.27-1.82$ & $<0.0001$ \\
\hline \multicolumn{6}{|l|}{ Race } \\
\hline White & 480 (93.9) & 35551 (91.6) & 1 & Reference & \\
\hline Black & $12(2.3)$ & $1953(5.0)$ & 2.20 & $1.24-3.90$ & 0.0072 \\
\hline \multicolumn{6}{|l|}{ Insurance } \\
\hline Uninsured & $12(2.3)$ & 1309 (3.4) & 1 & Reference & \\
\hline Government & $159(31.1)$ & $16039(41.3)$ & 0.92 & $0.51-1.67$ & 0.79 \\
\hline Private & $331(64.8)$ & $20989(54.1)$ & 0.58 & $0.33-1.04$ & 0.066 \\
\hline \multicolumn{6}{|l|}{ Income } \\
\hline$<48,000$ & $144(28.2)$ & $13295(34.3)$ & 1 & Reference & \\
\hline$\geq 48,000$ & $364(71.2)$ & $24677(63.6)$ & 0.73 & $0.60-0.89$ & 0.0018 \\
\hline \multicolumn{6}{|l|}{ Facility } \\
\hline Community & $11(2.2)$ & 1914 (4.9) & 1 & Reference & \\
\hline Comprehensive Community & $96(18.8)$ & $14059(36.2)$ & 0.85 & $0.45-1.57$ & 0.59 \\
\hline Academic & $368(72.0)$ & $20652(53.2)$ & 0.32 & $0.18-0.59$ & .0002 \\
\hline \multicolumn{6}{|l|}{ Population } \\
\hline Metro & $444(86.9)$ & 30847 (79.5) & 1 & Reference & \\
\hline Urban & $47(5.8)$ & $5647(14.6)$ & 1.73 & $1.28-2.34$ & 0.0004 \\
\hline Rural & $9(1.8)$ & $708(1.8)$ & 1.13 & $0.58-2.20$ & 0.71 \\
\hline \multicolumn{6}{|l|}{ Distance to facility } \\
\hline$\leq 12$ miles & $224(43.8)$ & $18746(48.3)$ & 1 & Reference & \\
\hline$>12$ miles & $284(55.6)$ & $19240(49.6)$ & 0.81 & $0.68-0.97$ & 0.019 \\
\hline \multicolumn{6}{|l|}{ Comorbid (Charlson-Deyo) } \\
\hline 0 & $394(77.1)$ & $29399(75.8)$ & 1 & Reference & \\
\hline 1 & 76 (14.9) & $6018(15.5)$ & 1.06 & $0.83-1.36$ & 0.64 \\
\hline 2 or higher & $41(8.0)$ & $3389(8.7)$ & 1.1 & $0.80-1.53$ & 0.53 \\
\hline \multicolumn{6}{|l|}{ Years } \\
\hline $2004-2006$ & $32(6.3)$ & $7943(20.5)$ & 1 & Reference & \\
\hline $2007-2009$ & $23(4.5)$ & $10076(26.0)$ & 1.76 & $1.03-3.02$ & 0.038 \\
\hline $2010-2012$ & 105 (20.5) & 12003 (30.9) & 0.46 & $0.31-0.68$ & 0.0001 \\
\hline $2013-2014$ & $351(68.7)$ & $8784(22.6)$ & 0.10 & $0.07-0.15$ & $<0.0001$ \\
\hline
\end{tabular}

LEGEND: OR, odds ratio. $\mathrm{Cl}$, confidence interval 
Table 3. Multivariable Cox Proportional Hazards Models for Overall Survival in Patients Receiving Immunotherapy for GBM.

\begin{tabular}{|c|c|c|}
\hline Significant Characteristic & Hazard of Death $(95 \% \mathrm{Cl})$ & $\mathbf{p}$ \\
\hline & $\begin{array}{l}\text { Cox Model without } \\
\text { Propensity Score }\end{array}$ & \\
\hline \multicolumn{3}{|l|}{ Age } \\
\hline$\leq 60$ & Reference & \\
\hline$>60$ & $1.3891(1.3542-1.4250)$ & $<0.0001$ \\
\hline \multicolumn{3}{|l|}{ Sex } \\
\hline Male & Reference & \\
\hline Female & $0.8858(0.8668-0.9053)$ & $<0.0001$ \\
\hline \multicolumn{3}{|l|}{ Insurance } \\
\hline Not Insure & Reference & \\
\hline Government & $0.9713(0.9125-1.0340)$ & 0.3618 \\
\hline Private Payer & $1.2101(1.1798-1.2411)$ & $<0.0001$ \\
\hline Unknown & $1.1204(1.0163-1.2350)$ & 0.0223 \\
\hline \multicolumn{3}{|l|}{ Comorbidity Score } \\
\hline 0 & Reference & \\
\hline 1 & $1.1256(1.0928-1.1595)$ & $<0.0001$ \\
\hline$\geq 2$ & $1.2510(1.2048-1.2990)$ & $<0.0001$ \\
\hline \multicolumn{3}{|l|}{ Race } \\
\hline White & Reference & \\
\hline African American & $0.8785(0.8346-0.9247)$ & $<0.0001$ \\
\hline Other & $0.8119(0.7628-0.8642)$ & $<0.0001$ \\
\hline \multicolumn{3}{|l|}{ Years } \\
\hline 2004-06 & Reference & \\
\hline 2007-09 & $0.9452(0.9169-0.9744)$ & 0.0003 \\
\hline $2010-12$ & $0.8775(0.8516-0.9041)$ & $<0.0001$ \\
\hline 2013-15 & $0.8302(0.8025-0.8589)$ & $<0.0001$ \\
\hline \multicolumn{3}{|l|}{ Income } \\
\hline$<38,000$ & Reference & \\
\hline $38,000-47,999$ & $1.0144(0.9745-1.0558)$ & 0.4853 \\
\hline $48,000-62,999$ & $0.9478(0.9205-0.9760)$ & 0.0003 \\
\hline$\geq 63,000$ & $0.8847(0.8556-0.9148)$ & $<0.0001$ \\
\hline \multicolumn{3}{|l|}{ Facility Type } \\
\hline Community Cancer Program & Reference & \\
\hline Comprehensive Cancer Program & $0.9545(0.9082-1.0041)$ & 0.0719 \\
\hline Academic/Research Program & $0.8590(0.8175-0.9027)$ & $<0.0001$ \\
\hline \multicolumn{3}{|l|}{ Education } \\
\hline$\geq 29 \%$ & Reference & \\
\hline 20 to 28.9 & $1.0523(1.0127-1.0934)$ & 0.0092 \\
\hline 14 to 19.9 & $1.0542(1.0139-1.0960)$ & 0.0079 \\
\hline$<14$ & $1.0477(1.0020-1.0956)$ & 0.0408 \\
\hline \multicolumn{3}{|l|}{ Immunotherapy } \\
\hline No Immunotherapy & Reference & \\
\hline Received Immunotherapy & $0.9142(0.8271-1.0104)$ & 0.0789 \\
\hline
\end{tabular}

Note: Education is quartiles of the percentage of persons with less than a high school education in the patients' residence census tract. Income is median household income in the patients' residence census tract.

included was then run to determine predictors of outcome (excluding those factors used to generate propensity score). On propensity matched analysis, overall survival was 18 versus 17 months in patients receiving and not receiving IMT, respectively $(\mathrm{p}=0.15)$. Higher comorbidity, lower
Table 4: Multivariable Cox Proportional Hazards Models for Overall Survival in Patients Receiving Immunotherapy for GBM with Propensity Score.

\begin{tabular}{|l|l|c|}
\hline Significant Characteristic & \multicolumn{1}{|c|}{ Hazard of Death $(95 \%$ Cl) } & p \\
\hline Cox Model with Propensity & & \\
\hline Score & & \\
\hline 0 & Reference & \\
\hline 1 & $1.2198(1.1844-1.2563)$ & $<0.0001$ \\
\hline 2 & $1.3302(1.2813-1.3810)$ & $<0.0001$ \\
\hline Income & & \\
\hline$<38,000$ & Reference & \\
\hline $38,000-47,999$ & $1.0503(1.0210-1.0805)$ & 0.0007 \\
\hline $48,000-62,999$ & $0.9870(0.9522-1.0230)$ & 0.4728 \\
\hline$\geq 63,000$ & $0.9282(0.9057-0.9513)$ & $<0.0001$ \\
\hline Sex & & \\
\hline Male & Reference & \\
\hline Female & $0.9046(0.8851-0.9244)$ & $<0.0001$ \\
\hline & & \\
\hline
\end{tabular}

income, and male gender were independent predictors of poorer survival on propensity matched multivariable analysis as shown in Table 4.

\section{Discussion}

GBM is the most common primary CNS malignancy with a highly aggressive nature. Despite advances over the last decade in the management of other disease sites, clinically significant therapeutic advances in GBM have been sparse. As almost all cases tend to recur and result in death, many attempts have been made unsuccessfully to improve outcomes over the past decade. While IMT has provided hope in other malignancies, our results show that although IMT usage in GBM therapy has been increasing since 2009, no survival benefit is offered.

Recently, IMT utilization has improved oncologic outcomes in a variety of hematopoietic and nonhematopoietic malignancies, resulting in the modification of long-standing treatment paradigms. As a result, this led to exploration of the role of IMT in upfront treatment of GBM. With the known challenges of therapy penetrance across the BBB and drug related toxicities, particularly perilesional edema, intralesional hemorrhage and necrosis, multiple studies have taken aim at improving outcomes in primary brain malignancies with systemic targeted and immunotherapy. Well known to be highly vascularized tumors, initial thought was for the malignancy to be responsive to bevacizumab (BV), a monoclonal antibody against vascular endothelial growth factor (anti-VEGF). In multiple prospective phase 2 and retrospective trials analyzing bevacizumab efficacy on recurrent GBM, there was found to be up to a 35\% increase in progression free survival $(\mathrm{PFS})^{18}$. With this improvement seen in salvage therapy, its efficacy was quickly queried in the upfront setting. Lai et al subsequently enrolled 70 patients with newly diagnosed GBM and combined BV with upfront 
radiotherapy and TMZ and found improvement in PFS but no change in overall survival ${ }^{19}$. Similarly, Chinot et al examined intravenous bevacizumab with radiotherapy and TMZ followed by maintenance BV and TMZ in the upfront management of 921 patients with new GBM. Although their results also showed improved PFS and maintenance of baseline quality of life and performance status, they showed an increased incidence in adverse effects and no significant increase in survival when compared to placebo ${ }^{20}$. Next, as pembrolizumab (antiPD1 immunotherapy) offered groundbreaking results for malignancies of lung primary and malignant melanoma, its value was tested in recurrent primary CNS tumors and found to have no clinical or histologic efficacy in a small series conducted on 22 patients. In this study, virtually all 22 patients showed tumor progression and median OS was a mere 2.6 months for adults and 3.2 months for children ${ }^{7}$. This study coincides particularly well with CheckMate-143, which as previously annotated showed no improvement in survival when nivolumab was chosen over BV in recurrent GBM. Nivolumab continues to be evaluated in both CheckMate-498, where its efficacy in combination with radiotherapy is being compared against TMZ in combination with radiotherapy in newly diagnosed MGMT-unmethylated GBM, and its companion phase 3 trial, CheckMate-548, where evaluation of the addition of upfront nivolumab to TMZ and radiotherapy in newly diagnosed MGMT-methylated GBM is ongoing.

While these results emerge, many trials continue analyzing individual checkpoint inhibitors but results remain either limited or disappointing. However, other innovations in immune therapy continue to arise, including different delivery mechanisms to the tumor site, targeted therapies, dendritic cell vaccines, injecting antibodies directly into the tumor, and recombinant immunotoxins. Although most clinical trials have not officially resulted, there is some belief that combining these different modalities of immunotherapy to work synergistically is the future of GBM management ${ }^{21}$. With this in mind, numerous trials remain ongoing and more are enrolling patients each day.

When compared with previous studies analyzing upfront usage of IMT in GBM management, this NCDB analysis corroborates appropriately. Although we initially found an increase in overall survival on univariable analysis, this finding was no longer significant once propensity matching was performed. Interestingly, IMT use in the years 2007-2009 was low and subsequently skyrocketed which contradicts previously annotated phase 2 trials which initially showed improvement in PFS for BV salvage therapy without altering OS. It seems as though clinicians latched onto initial numbers and began using IMT in upfront treatment, and despite RTOG 0825, a clinical trial analyzing upfront BV usage, quickly showing no benefit in overall survival, use of IMT, in this case targeted therapy, continued to rise. Another possible reason for this rapid surge are the increased number of previously annotated clinical trials testing various combinations of immunomodulating therapies that remain ongoing. With this in mind we remain but must recommend against the use of IMT in the upfront management of GBM outside the confines of a clinical trial.

As is typical with these types of analyses, this study was limited by the data provided in the NCDB due to its retrospective nature and inherent selection bias. Compounding this, the NCDB lacks information on toxicity, local failure, which systemic therapeutic agent(s) were used and the number of cycles completed, and KPS and MGMT status were not incorporated until 2010, all of which play an important role in management and ultimately outcome. Also, the specific IMT used is not documented and the category in the NCDB encompasses a broad range of IMT as described in the methods, included targeted therapies. Additionally, salvage therapy is not recorded in the NCDB which plays an important role in GBM survival given the high rates of recurrence. Finally, patients whose clinician substituted IMT for chemotherapy off-label were not recorded in this study as they did not meet selection criteria.

\section{Conclusion}

The use of IMT in the upfront treatment of GBM is associated with similar survival as its absence. Therefore, these should be limited to use within the confines of ongoing clinical trials.

\section{Compliance with Ethical Standards}

\section{Funding}

This study received no funding.

\section{Conflict of Interest}

No author present on this article has any conflicts of interest.

\section{Ethical approval}

This study does not contain any studies with human participant performed by any of the authors.

\section{References}

1. Preusser M, Lim M, Hafler DA, et al. Prospects of immune checkpoint modulators in the treatment of glioblastoma. Nat Rev Neurol. 2015; 11(9): 504-514. doi:10.1038/nrneurol.2015.139

2. Stupp R, Mason WP, van den Bent MJ, et al. Radiotherapy plus Concomitant and Adjuvant Temozolomide for Glioblastoma. N Engl J Med. 2005; 352(10): 987-996. doi:10.1056/NEJMoa043330

3. Westphal M, Hilt DC, Bortey E, et al. A phase 3 trial of local chemotherapy with biodegradable carmustine (BCNU) wafers 
(Gliadel wafers) in patients with primary malignant glioma. Neuro Oncol. 2003; 5(2): 79-88. doi:10.1093/neuonc/5.2.79

4. Chatzikonstantinou G, Zamboglou N, Archavlis E, et al. CT-guided interstitial HDR-brachytherapy for recurrent glioblastoma multiforme: a 20-year single-institute experience. Strahlenther Onkol. 2018; 194(12): 1171-1179. doi:10.1007/s00066-018-1358-3

5. Stupp R, Taillibert S, Kanner A, et al. Effect of Tumor-Treating Fields Plus Maintenance Temozolomide vs Maintenance Temozolomide Alone on Survival in Patients With Glioblastoma: A Randomized Clinical Trial. JAMA. 2017; 318(23): 2306-2316. doi:10.1001/ jama.2017.18718

6. Cohen J V, Kluger HM. Systemic Immunotherapy for the Treatment of Brain Metastases. Front Oncol. 2016; 6: 49. doi:10.3389/ fonc.2016.00049

7. Blumenthal DT, Yalon M, Vainer GW, et al. Pembrolizumab: first experience with recurrent primary central nervous system (CNS) tumors. J Neurooncol. 2016; 129(3): 453-460. doi:10.1007/s11060016-2190-1

8. Hasan S, Renz P, Turrisi A, et al. Dose escalation and associated predictors of survival with consolidative thoracic radiotherapy in extensive stage small cell lung cancer (SCLC): A National Cancer Database (NCDB) propensity-matched analysis. Lung Cancer. 2018; 124: 283-90.

9. Hasan S, Renz P, Wegner RE, et al. Microsatellite Instability (MSI) as an Independent Predictor of Pathologic Complete Response (PCR) in Locally Advanced Rectal Cancer: A National Cancer Database (NCDB) Analysis. Ann Surg. 2018.

10. Verma V, Simone CB 2nd, Lin C. Human papillomavirus and nasopharyngeal cancer. Head Neck 2018; 40: 696-706.

11. Ryckman JM, Kusi Appiah A, Simone CB 2nd, et al. Treatment approaches for nasopharyngeal adenoid cystic carcinoma. Acta Oncol 2018; doi: 10.1080/0284186X.2018.1426878.
12. Verma V, Surkar SM, Moreno AC, et al. Practice patterns and outcomes of chemoradiotherapy versus radiotherapy alone for older patients with nasopharyngeal cancer. Cancer Med. 2018; doi: 10.1002/ cam4.1290.

13. Ryckman JM, Lin C, Simone CB 2nd, et al. Patterns of Care for Stage IA Cervical Cancer: Use of Definitive Radiation Therapy Versus Hysterectomy. Int J Gynecol Cancer. 2018; 28: 773-781.

14. Deyo RA, Cherkin DC, Ciol MA. Adapting a clinical comorbidity index for use with ICD-9-CM administrative databases. J Clin Epidemiol. 1992; 45(6): 613-9.

15. Meier ELKaP. Nonparametric Estimation from Incomplete Observations. Journal of the American Statistical Association. 1958; 53(282): 457-81.

16. Cox DR. Regression Models and Life- Tables. Journal of the Royal Statistical Society. 1972; 34(2): 187-220.

17. D'Agostino RB, Jr. Propensity score methods for bias reduction in the comparison of a treatment to a non-randomized control group. Stat Med. 1998; 17(19): 2265-81.

18. Chamberlain MC. Bevacizumab for the treatment of recurrent glioblastoma. Clin Med Insights Oncol. 2011; 5: 117-129. doi:10.4137/ CMO.S7232

19. Lai A, Tran A, Nghiemphu PL, et al. Phase II study of bevacizumab plus temozolomide during and after radiation therapy for patients with newly diagnosed glioblastoma multiforme. J Clin Oncol. 2011; 29(2): 142-148. doi:10.1200/JC0.2010.30.2729

20. Chinot OL, Wick W, Mason W, et al. Bevacizumab plus radiotherapytemozolomide for newly diagnosed glioblastoma. N Engl J Med. 2014 370(8): 709-722. doi:10.1056/NEJMoa1308345

21. Patel MA, Pardoll DM. Concepts of immunotherapy for glioma. J Neurooncol. 2015; 123(3): 323-30. 